\title{
Results of the feasibility test of the Practical Approach to Lung Health in the Syrian Arab Republic
}

F. Me'emary, ${ }^{1}$ S-E. Ottmani, ${ }^{2}$ A. Pio, ${ }^{3}$ S. Baghdadi, ${ }^{4}$ G. Assafin, ${ }^{5}$ M. Koraym,${ }^{1}$ M. Saleh,${ }^{6}$ H. Bashour, ${ }^{7}$ A. Seita ${ }^{4}$ and L. Blanc ${ }^{2}$

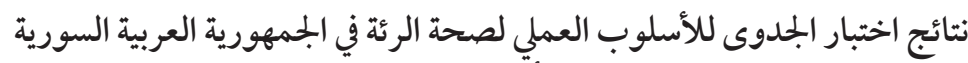

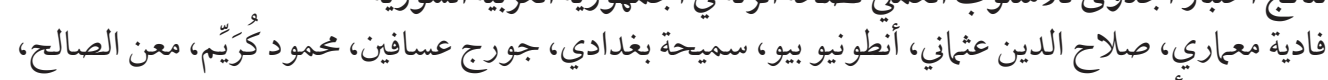

$$
\begin{aligned}
& \text { هيام بشور، أكيهيرو سيتا، ليوبولد بلانلكانك }
\end{aligned}
$$

ABSTRACT We assessed implementation of the Practical Approach to Lung Health (PAL) in primary care facilities in the Syrian Arab Republic and its short-term impact on respiratory care in patients aged $5+$ years. After training on PAL for 76 general practitioners in 75 health centres, referrals and sputum smear examinations for patients increased. The mean number of drugs prescribed per patient decreased by $14.8 \%$ and that of antibiotics by $33.3 \%$, while prescriptions for inhaled medications increased. The mean cost of drug prescriptions fell by $26.2 \%$.

Résultats du test de faisabilité de l'approche pratique de la santé respiratoire en République arabe syrienne

RÉSUMÉ Nous avons évalué la mise en œuvre de l'approche pratique de la santé respiratoire (APSR) dans les services de soins de santé primaires de la République arabe syrienne et ses effets à court terme sur les soins respiratoires chez les patients âgés de 5 ans et plus. Après la formation à l'APSR dispensée à 76 médecins généralistes dans 75 centres de santé, le nombre d'orientations de patients vers des services spécialisés et d'examens de frottis de crachats a progressé. Le nombre moyen de médicaments prescrits par patient a diminué de $14,8 \%$ et celui des antibiotiques de $33,3 \%$, alors que les prescriptions de médicaments inhalés ont augmenté. Le coût moyen des prescriptions de médicaments a chuté de $26,2 \%$.

\footnotetext{
${ }^{1}$ Ministry of Health, Damascus, Syrian Arab Republic.

${ }^{2}$ Stop TB Department, World Health Organization, Geneva, Switzerland (Correspondence to S-E. Ottmani: ottmanis@who.int).

${ }^{3}$ Senior Consultant, Mar Del Plata, Argentina.

${ }^{4}$ Stop TB Unit, Department of Communicable Diseases, World Health Organization Regional Office for the Eastern Mediterranean, Cairo, Egypt.

${ }^{5}$ Syrian Chest Disease Association, Damascus, Syrian Arab Republic.

${ }^{6}$ Private Chest Clinic, Damascus, Syrian Arab Republic.

${ }^{7}$ Department of Epidemiology, Damascus University School of Medicine, Damascus, Syrian Arab Republic. Received: 01/7/08; accepted: 18/11/08
} 


\section{Introduction}

Respiratory illness is common throughout the world and are often the leading reason for seeking care in primary health care (PHC) settings. Surveys carried out by the World Health Organization (WHO) in 9 developing countries showed that approximately $20 \%$ of patients attend PHC facilities for respiratory symptoms [1]. Within the framework of the Stop TB Strategy, WHO has developed the Practical Approach to Lung Health (PAL) which aims to improve the quality of management of respiratory patients [2]. It is a patient-centred approach to diagnosis and treatment of common respiratory illnesses encountered in PHC settings. It promotes a symptom-based and integrated approach to management, and seeks to standardize service delivery through the development and implementation of clinical guidelines. PAL is intended to ensure coordination among different levels of health care and between tuberculosis (TB) control programmes and general health services in order to improve the efficiency of respiratory case management within the district health system [3].

Successful implementation of PAL in a country needs to be adapted to the national health policy and priorities as well as to the existing human and logistic resources [3]. It also needs to follow a stepwise process [4]. In October 2003, the National TB Programme (NTP) of the Syrian Arab Republic, in collaboration with WHO, initiated the process of adoption of PAL.

Further to a field assessment jointly carried out by WHO and the Syrian Ministry of Health in March 2004, a national working group on PAL was established and an agenda for development and implementation of the approach was adopted. The first activity of the working group was to develop guide- lines and training materials for PAL in line with WHO guidance. The next step was to test the feasibility of implementation of the PAL guidelines and assess the short-term impact on the clinical practices of general practitioners (GPs) in PHC facilities. The feasibility test was based on the comparison between a baseline survey, before training GPs about PAL, and an impact survey, done shortly after training.

The objective of this paper is to present and discuss the findings of this feasibility test, taking into account the limitations of the methodology used and the PAL experience obtained so far in other countries.

\section{Methods}

The baseline and impact surveys were conducted according to the protocol specified by WHO for testing the feasibility of PAL [5].

\section{Study setting and sample}

The baseline and impact surveys were carried out in the same PHC facilities located in 3 governorates and involved the same GPs. The governorates of Daraa, Homs and Latakia were selected because of the availability of qualified health personnel and the technical and logistic capacity to monitor the quality of data collection, compile these data and supervise the health staff involved in the surveys.

A total of 75 PHC facilities were selected as follows: 18 in Daraa, 29 in Homs and 28 in Latakia. These PHC facilities were selected because of their level of involvement in ensuring PHC service delivery to the community and easy access for patients to the TB laboratory and referral levels. In each of the 75 PHC facilities, at least 1 GP was enrolled on a voluntary basis in the feasibility test and in 1 facility in Daraa 2 were

المجلة الصحية لشرق المتوسط، منظمة الصحة العالمية، المجلد الخنامس عشر، العدد ب، 9 +. 
enrolled, making a total of 76 GPs involved in both surveys. Each GP was considered a data collection site.

\section{Data collection methods}

Participant enrolment

The eligibility criteria for data collection were: age $\geq 5$ years and had sought care for $\geq 1$ respiratory symptom in any of the selected PHC facilities during the survey period. Both surveys used the same definition of a TB suspect as the NTP, i.e. any patient with productive cough $\geq 15$ days. Other respiratory conditions were classified according to the categories proposed in the study protocol, according to the $I C D-10[6]$.

During the data collection periods all eligible respiratory patients who sought care in the 75 selected PHC facilities were seen by the 76 GPs enrolled in the study.

\section{Tools}

The data were collected in the same manner in both surveys. For each eligible patient the GP recorded: identification number, description of clinical symptoms, demographic characteristics, initial diagnosis and management decision. For each patient, the same identification number was specified in the forms used for requesting complementary investigations, patient drug prescriptions, treatment cards and any other patient health documents. This number was used to collect follow-up data on patients. If a patient was prescribed drugs, a copy of that prescription was kept on the data collection site.

Furthermore, in each of the 2 surveys, data were collected on the number of patients who visited the PHC facility for any reason, recorded by sex and age. Specific information was also collected from the TB laboratories and TB and chest disease clinics to assess the follow-up of each TB suspect identified in the survey registers.

\section{Baseline survey}

The 76 GPs were first trained to collect the data on the study register and the other forms in order to standardize the data collection process. Data collection for the baseline survey was carried out from 11 to 15 December 2005. Follow-up information was collected during the 30 days after registration, as specified in the study protocol.

\section{Training on PAL guidelines}

From 8 to 12 January 2006, the 76 GPs involved in the baseline survey were trained on the use of the locally adapted PAL guidelines. The training was carried out by the national working group members and the study supervisors.

\section{Impact survey}

From 22 to 26 January 2006, the impact survey was carried out in the same 75 PHC facilities, involving the same 76 GPs who had received training on PAL. The process of data collection was the same as forthe baseline survey. As well as applying the knowledge received during training, GPs could use the PAL guidelines to manage respiratory patients during this second survey. The 30-day follow-up period for the impact survey ended on 23 February 2006.

\section{Monitoring system}

The national working group coordinated the various activities inherent in the feasibility test, ensured the logistics needed, supported the supervisory activities and checked the quality of data collection. Moreover, 3 groups (1 for each governorate) of 5 study supervisors each were established. Each group ensured that GPs were trained on data collection as well as on PAL guidelines and was responsible for the supervision and 
monitoring of the survey activities in the selected PHC facilities.

\section{Statistical analysis}

The analysis involved comparison of the distribution of the data on the variables in the baseline and impact surveys. The Student $t$-test was used for comparing 2 means and the chi-squared test for comparing proportions. The Fisher exact test was used when the expected number was below 5 in $2 \times 2$ contingency tables. The MannWhitney test was used to compare a nonnormal distribution of a variable between the baseline and impact surveys. A statistical difference was considered significant when $P$-value was below $5 \%$.
A data entry programme was adapted and developed specifically for these surveys, using Epi-Info, version 6.04b.

\section{Results}

\section{Characteristics of patients}

The baseline and impact surveys registered 1806 and 1099 respiratory patients respectively aged $5+$ years in the 75 selected PHC units, a $39.1 \%$ decrease between the surveys.

The proportion of respiratory patients among all outpatients of this age group was significantly different between the baseline survey (35.8\%) and the impact survey

\begin{tabular}{|c|c|c|c|c|c|}
\hline \multirow[t]{2}{*}{ Patient characteristic } & \multicolumn{2}{|c|}{ Baseline survey } & \multicolumn{2}{|c|}{ Impact survey } & \multirow[t]{2}{*}{$P$-value } \\
\hline & No. & $\%$ & No. & $\%$ & \\
\hline \multicolumn{6}{|l|}{ All conditions } \\
\hline Female & 3174 & 63.0 & 2864 & 63.5 & \multirow{3}{*}{$>0.05$} \\
\hline Male & 1865 & 37.0 & 1646 & 36.5 & \\
\hline Total & 5039 & 100.0 & 4510 & 100.0 & \\
\hline \multicolumn{6}{|l|}{ Respiratory symptoms } \\
\hline Female & 1004 & 55.6 & 613 & 55.8 & \multirow{3}{*}{$>0.05$} \\
\hline Male & 802 & 44.4 & 486 & 44.2 & \\
\hline Total & 1806 & 100.0 & 1099 & 100.0 & \\
\hline \multicolumn{6}{|l|}{$\begin{array}{l}\text { Respiratory symptoms/all } \\
\text { patients }\end{array}$} \\
\hline Female & $1004 / 3174$ & 31.6 & $613 / 2864$ & 21.4 & $<0.001$ \\
\hline Male & $802 / 1865$ & 43.0 & $486 / 1646$ & 29.5 & $<0.001$ \\
\hline \multirow[t]{2}{*}{ Total } & $1806 / 5039$ & 35.8 & $1099 / 4510$ & 24.4 & $<0.001$ \\
\hline & \multicolumn{2}{|c|}{ Mean (SD) } & \multicolumn{2}{|c|}{ Mean (SD) } & \\
\hline \multicolumn{6}{|l|}{ Age (years) } \\
\hline Female & \multicolumn{2}{|c|}{$23.9(17.6)^{a}$} & \multicolumn{2}{|c|}{$25.1(17.4)^{\mathrm{b}}$} & $>0.05$ \\
\hline Male & \multicolumn{2}{|c|}{$20.0(19.1)^{a}$} & \multicolumn{2}{|c|}{$22.5(18.8)^{b}$} & $<0.05$ \\
\hline Total & \multicolumn{2}{|c|}{$22.2(18.3)$} & \multicolumn{2}{|c|}{$24.0(18.0)$} & $<0.05$ \\
\hline
\end{tabular}

${ }^{a}$ Comparison of mean age of females and males in the baseline survey $(\mathrm{P}<0.001)$

${ }^{b}$ Comparison of mean age of females and males in the baseline survey $(P<0.05)$.

$S D=$ standard deviation.

المجلة الصحية لشرق المتوسط، منظمة الصحة العالمية، المجلد الخنامس عشر، العدد ب، 9 +. 
$508 \quad$ La Revue de Santé de la Méditerranée orientale, Vol. 15, №3, 2009

Table 2 Frequency of symptoms and signs, concomitant diseases and risk

factors among respiratory patients in the Syrian Arab Republic

\begin{tabular}{|c|c|c|c|c|c|}
\hline Characteristic & \multicolumn{2}{|c|}{$\begin{array}{c}\text { Baseline survey } \\
\quad(n=1806) \\
\text { Mean (SD) }\end{array}$} & \multicolumn{2}{|c|}{$\begin{array}{l}\text { Impact survey } \\
\quad(n=1099)^{a} \\
\text { Mean (SD) }\end{array}$} & P-value \\
\hline \multirow{2}{*}{ Duration of symptoms (days) } & \multicolumn{2}{|c|}{$3.7(4.6)^{b}$} & \multicolumn{2}{|c|}{$3.9(4.7)$} & $>0.05^{c}$ \\
\hline & No. & $\%$ & No. & $\%$ & \\
\hline Visits in previous month & 218 & 12.1 & 147 & 13.4 & $>0.05$ \\
\hline \multicolumn{6}{|l|}{ Sign/symptom } \\
\hline Cough & 936 & 51.8 & 496 & 45.2 & $<0.001$ \\
\hline Fever & 1025 & 56.8 & 551 & 50.2 & $<0.001$ \\
\hline Nasal discharge & 487 & 27.0 & 314 & 28.6 & $>0.05$ \\
\hline Sore throat & 508 & 28.1 & 298 & 27.2 & $>0.05$ \\
\hline Expectoration & 215 & 11.9 & 147 & 13.4 & $>0.05$ \\
\hline Shortness of breath & 166 & 9.2 & 122 & 11.1 & $>0.05$ \\
\hline Nasal obstruction & 32 & 1.8 & 9 & 0.8 & $<0.05$ \\
\hline Chest pain & 97 & 5.4 & 65 & 5.9 & $>0.05$ \\
\hline Wheezing & 82 & 4.5 & 55 & 5.0 & $>0.05$ \\
\hline Otalgia & 114 & 6.3 & 76 & 6.9 & $>0.05$ \\
\hline Sneezing & 28 & 1.6 & 15 & 1.4 & $>0.05$ \\
\hline Ear discharge & 8 & 0.4 & 8 & 0.7 & $>0.05$ \\
\hline Dysphonia & 20 & 1.1 & 14 & 1.3 & $>0.05$ \\
\hline Other symptoms & 353 & 19.5 & 291 & 26.5 & $<0.001$ \\
\hline \multicolumn{6}{|l|}{ Concomitant disease } \\
\hline Any & 177 & 9.8 & 77 & 7.0 & $<0.01$ \\
\hline Chronic respiratory disease & 57 & 3.2 & 25 & 2.3 & $>0.05$ \\
\hline Hypertension & 45 & 2.5 & 16 & 1.5 & $>0.05$ \\
\hline Coronary heart disease & 11 & 0.6 & 7 & 0.6 & $>0.05$ \\
\hline Diabetes & 36 & 2.0 & 13 & 1.2 & $>0.05$ \\
\hline Other diseases & 52 & 2.9 & 25 & 2.3 & $>0.05$ \\
\hline \multicolumn{6}{|l|}{ Risk factor } \\
\hline Any & 570 & 31.6 & 287 & 26.1 & $<0.01$ \\
\hline Smoking & 186 & 10.3 & 122 & 11.1 & $>0.05$ \\
\hline Alcohol & 1 & 0.1 & 0 & 0.0 & $>0.05^{d}$ \\
\hline Pregnancye & $11 / 457$ & 2.4 & $4 / 320$ & 1.3 & $>0.05$ \\
\hline Other risk factors & 357 & 19.8 & 160 & 14.5 & $<0.001$ \\
\hline
\end{tabular}

${ }^{a}$ For prevalence of symptoms $\mathrm{n}=1097$ because no information on symptoms was registered in 2 patients. ${ }^{b}$ Duration of symptoms not registered in 1 patient in the baseline survey. ${ }^{c}$ MannWhitney test; ${ }^{\prime}$ Fisher exact test. ${ }^{e}$ Denominator $=$ number of women of child-bearing age.

$S D=$ standard deviation .

(24.4\%) (Table 1) $(P<0.001)$. The proportion of female patients enrolled was higher than that of males in both surveys (Table 1). However, the percentage of respiratory patients as a proportion of all outpatients was higher in males than in females and declined with age in both sexes (data not shown).

The mean age of study participants was 22.2 years in the baseline and 24.0 years 
in the impact survey. In both surveys, the mean age was greater in females.

No significant differences were found between the 2 surveys in terms of: duration of symptoms before presentation; proportion of patients who visited any health facility at least once for respiratory symptoms within the last month; proportion of patients with existing chronic respiratory disease; and proportion of patients using tobacco at the time of the survey (Table 2). Cough, fever, nasal discharge, sore throat and expectoration were the most frequently reported symptoms in both surveys.

\section{Distribution of patients}

In general, there was no significant difference in the distribution of respiratory conditions between the 2 surveys. Acute respiratory infection accounted for $>90 \%$ of respiratory patients and chronic respiratory disease for $<10 \%$ (Table 3 ). The proportion of patients with asthma was $4.7 \%$ in both surveys, while for chronic obstruc-

\begin{tabular}{|c|c|c|c|c|}
\hline \multirow[t]{2}{*}{$\begin{array}{l}\text { Respiratory } \\
\text { condition }\end{array}$} & \multicolumn{2}{|c|}{$\begin{array}{c}\text { Baseline } \\
\text { survey } \\
(n=1806)\end{array}$} & \multicolumn{2}{|c|}{$\begin{array}{c}\text { Impact } \\
\text { survey } \\
(n=1099)\end{array}$} \\
\hline & No. & $\%$ & No. & $\%$ \\
\hline $\mathrm{ARI}$ & 1688 & 93.4 & 1006 & 91.5 \\
\hline AURI & 1258 & 69.7 & 739 & 67.2 \\
\hline ALRI & 417 & 23.1 & 251 & 22.8 \\
\hline Pneumonia & 13 & 0.7 & 16 & 1.5 \\
\hline CRD & 99 & 5.5 & 73 & 6.6 \\
\hline Asthma & 85 & 4.7 & 52 & 4.7 \\
\hline COPD & 12 & 0.7 & 17 & $1.5^{\star}$ \\
\hline Other condition & 19 & 1.1 & 20 & 1.8 \\
\hline
\end{tabular}

*P $<0.05$.

$A R I=$ acute respiratory infection; $A U R I=$ acute upper respiratory infection; $A L R I=$ acute lower respiratory infection (not pneumonia); $C R D=$ chronic respiratory disease; $C O P D=$ chronic obstructive pulmonary disease. tive pulmonary disease it was significantly higher in the impact survey $(1.5 \%)$ than in the baseline survey $(0.7 \%)(P<0.05)$.

The overall referral rate increased from $4.5 \%$ in the baseline survey to $6.6 \%$ in the impact survey $(P<0.05)$, mainly due to the significant increase in referral for hospitalization $(0.3 \%$ to $1.2 \%)(P<0.05)$ (Table 4$)$.

\section{Detection of TB}

The proportion of respiratory patients in whom sputum smear examinations were requested increased significantly from $0.2 \%$ in the baseline survey to $1.9 \%$ in the impact survey (Table 5). The increase among patients who had respiratory symptoms for $\geq 15$ days rose from $4.5 \%$ to $17.4 \%$. Among all respiratory patients, the proportion of those who underwent sputum smear examinations significantly increased from $0.2 \%$ in the baseline survey to $1.5 \%$ in the impact survey. No smear-positive TB case was identified in either survey; however, 1 case of cervical lymph TB was diagnosed in the impact survey.

\section{Drug prescription patterns}

Of the respiratory patients $96.7 \%$ received a drug prescription in the baseline survey and $94.4 \%$ in the impact survey (Table 6). The mean number of drugs prescribed per patient decreased by $14.8 \%$ in the impact survey and the mean number of drugs prescribed per patient who received a prescription decreased by $14.3 \%$.

Among patients who received a drug prescription, the proportion who were prescribed antibiotics decreased by $27.4 \%$ in the impact study, while the number of antibiotics per patient prescribed drugs decreased by $33.3 \%$. Antibiotic prescriptions decreased in all categories of respiratory condition. Among patients who were prescribed antibiotics, the proportion who received amoxicillin or penicillin increased

المجلة الصحية لشرق المتو سط، منظمة الصحة العالمية، المجلد الخامس عشر، العدد ب، 9 +. 


\begin{tabular}{|c|c|c|c|c|c|c|}
\hline \multirow{2}{*}{$\begin{array}{l}\text { Referral and } \\
\text { complementary } \\
\text { investigations }\end{array}$} & \multicolumn{2}{|c|}{$\begin{array}{l}\text { Baseline survey } \\
\quad(n=1806)\end{array}$} & \multicolumn{2}{|c|}{$\begin{array}{l}\text { Impact survey } \\
\quad(n=1099)\end{array}$} & \multirow[t]{2}{*}{$\begin{array}{c}\% \\
\text { change }^{a}\end{array}$} & \multirow[t]{2}{*}{$P$-value } \\
\hline & No. & $\%$ & No. & $\%$ & & \\
\hline Overall referrals & 82 & 4.5 & 72 & 6.6 & +46.7 & $<0.05$ \\
\hline Hospitalization & 5 & 0.3 & 13 & 1.2 & +300.0 & $<0.05$ \\
\hline Specialist & 16 & 0.9 & 14 & 1.3 & +44.4 & $>0.05$ \\
\hline Laboratory tests $^{b}$ & 15 & 0.8 & 3 & 0.3 & -62.5 & $>0.05$ \\
\hline Chest X-ray & 39 & 2.2 & 31 & 2.8 & +27.3 & $>0.05$ \\
\hline Other tests & 15 & 0.8 & 7 & 0.6 & -25.0 & $>0.05$ \\
\hline
\end{tabular}

${ }^{a}$ Between impact and baseline surveys.

${ }^{b}$ Laboratory tests other than tuberculosis sputum smear examination.

significantly, while those who were prescribed cephalosporins, oxacillin, lincomycin and quinolones decreased significantly in the impact survey (data not shown).

Among respiratory patients who were prescribed any drug, the prescription of bronchodilator drugs was similar in both surveys $(7.5 \%$ and $7.2 \%$ ) (Table 6 ). However, prescriptions significantly increased for inhaled $\beta$-agonists (1.4\% to $3.9 \%)$ and decreased for the other $\beta$-agonist forms and for theophylline in the impact survey (data not shown). The proportion of patients prescribed corticosteroids drugs increased nonsignificantly, the proportion prescribed inhaled corticosteroids increased significantly, $0.2 \%$ to $1.4 \%(P<0.001)$.
For respiratory patients who received a drug prescription, the proportion who were prescribed expectorants, aspirin, antihistamines and vitamins significantly decreased in the impact survey in contrast to the significant increase in the proportion who were prescribed paracetamol $(57.6 \%$ to $65.2 \%)$.

\section{Cost of drug prescriptions}

The mean cost of the prescription of any drug per patient who was prescribed drugs decreased significantly $(-26.2 \%)$ in the impact survey $(P<0.001)$ (Table 7$)$. This decrease was significant in patients with acute upper respiratory infection as well as in those with acute lower respiratory infec-

Table 5 Sputum smear examinations (SSE) according to symptom duration in the baseline and impact surveys in the Syrian Arab Republic

\begin{tabular}{|c|c|c|c|c|c|c|c|c|c|c|c|c|}
\hline \multirow{3}{*}{$\begin{array}{l}\text { Duration of } \\
\text { respiratory } \\
\text { symptoms }\end{array}$} & \multicolumn{6}{|c|}{ Baseline survey } & \multicolumn{6}{|c|}{ Impact survey } \\
\hline & \multicolumn{2}{|c|}{ Total } & \multicolumn{2}{|c|}{$\begin{array}{c}\text { SSE } \\
\text { requested }\end{array}$} & \multicolumn{2}{|c|}{$\begin{array}{c}\text { SSE } \\
\text { performed }\end{array}$} & \multicolumn{2}{|c|}{ Total } & \multicolumn{2}{|c|}{$\begin{array}{c}\text { SSE } \\
\text { requested }\end{array}$} & \multicolumn{2}{|c|}{$\begin{array}{c}\text { SSE } \\
\text { performed }\end{array}$} \\
\hline & No. & $\%$ & No. & $\%$ & No. & $\%$ & No. & $\%$ & No. & $\%$ & No. & $\%$ \\
\hline$<15$ days & 1739 & 96.3 & 1 & 0.06 & - & - & $1053^{*}$ & 95.8 & 13 & $1.2^{\star \star \star}$ & - & - \\
\hline$\geq 15$ days & 66 & 3.7 & 3 & 4.5 & - & - & $46^{*}$ & 4.2 & 8 & $17.4^{\star \mathrm{a}}$ & - & - \\
\hline Total & $1805^{b}$ & 100.0 & 4 & 0.2 & 3 & 0.2 & 1099 & 100.0 & 21 & $1.9^{\star \star \star}$ & 16 & $1.5^{\star \star \star}$ \\
\hline
\end{tabular}

${ }^{a}$ Fisher exact test; ${ }^{b}$ Duration of symptoms was not registered for 1 patient in the baseline survey. ${ }^{*} \mathrm{P}>0.05 ;{ }^{* * *} \mathrm{P}<0.001$. 
Table 6 Drug prescription among respiratory patients in the baseline and impact surveys in the Syrian Arab Republic

\begin{tabular}{|c|c|c|c|c|c|c|}
\hline Drug prescription & \multicolumn{2}{|c|}{$\begin{array}{l}\text { Baseline survey } \\
\text { Mean (SD) }\end{array}$} & \multicolumn{2}{|c|}{$\begin{array}{l}\text { Impact survey } \\
\text { Mean (SD) }\end{array}$} & $\%$ change & $P$-value \\
\hline No. drugs prescribed per patient & \multicolumn{2}{|c|}{$2.70(1.04)$} & \multicolumn{2}{|c|}{$2.30(0.93)$} & -14.8 & $<0.001$ \\
\hline $\begin{array}{l}\text { No. drugs per patient prescribed } \\
\text { drugs }\end{array}$ & \multicolumn{2}{|c|}{$2.80(0.93)$} & \multicolumn{2}{|c|}{$2.40(0.76)$} & -14.3 & $<0.001$ \\
\hline $\begin{array}{l}\text { No. antibiotics prescribed per } \\
\text { patient prescribed drugs }\end{array}$ & \multicolumn{2}{|c|}{$1.11(0.64)$} & \multicolumn{2}{|c|}{$0.74(0.65)$} & -33.3 & $<0.001$ \\
\hline \multicolumn{7}{|l|}{ No. antibiotics per patient } \\
\hline & No. & $\%$ & No. & $\%$ & & \\
\hline $\begin{array}{l}\text { No. patients prescribed drugs/ } \\
\text { total no. patients }\end{array}$ & $1747 / 1806$ & 96.7 & $1038 / 1099$ & 94.4 & -2.4 & $<0.01$ \\
\hline $\begin{array}{l}\text { No. patients prescribed antibiotics/ } \\
\text { no. patients prescribed drugs }\end{array}$ & $1511 / 1747$ & 86.5 & $652 / 1038$ & 62.8 & -27.4 & $<0.001$ \\
\hline $\begin{array}{l}\text { No. patients prescribed antibiotics/ } \\
\text { no. patients prescribed drugs for: }\end{array}$ & & & & & & \\
\hline AURI & $1057 / 1222$ & 86.5 & $467 / 705$ & 66.2 & -23.5 & $<0.001$ \\
\hline ALRI & $388 / 419$ & 92.6 & $157 / 252$ & 62.3 & -32.7 & $<0.001$ \\
\hline CRD & $59 / 93$ & 63.4 & $23 / 69$ & 33.3 & -47.5 & $<0.001$ \\
\hline Other & $7 / 13$ & 53.8 & $5 / 12$ & 41.7 & -22.5 & $>0.05$ \\
\hline $\begin{array}{l}\text { No. patients prescribed a specific } \\
\text { medication/no. prescribed drugs: }\end{array}$ & & & & & & \\
\hline Any bronchodilator & 131 & 7.5 & 75 & 7.2 & -4.0 & $>0.05$ \\
\hline Inhaled $\beta$-agonist & 25 & 1.4 & 41 & 3.9 & +178.6 & $<0.001$ \\
\hline Any corticosteroid & 55 & 3.1 & 35 & 3.4 & +9.7 & $>0.05$ \\
\hline Inhaled corticosteroid & 3 & 0.2 & 15 & 1.4 & +600.0 & $<0.001$ \\
\hline Expectorant & 518 & 29.7 & 257 & 24.8 & -16.5 & $<0.01$ \\
\hline Antitussive & 349 & 20.0 & 198 & 19.1 & -4.5 & $>0.05$ \\
\hline Nasal decongestant & 672 & 38.5 & 378 & 36.4 & -5.5 & $>0.05$ \\
\hline Aspirin & 40 & 2.3 & 9 & 0.9 & -60.9 & $<0.01$ \\
\hline Paracetamol & 1006 & 57.6 & 676 & 65.2 & +13.2 & $<0.001$ \\
\hline Antihistamine & 699 & 40.0 & 359 & 34.6 & -13.5 & $<0.01$ \\
\hline Vitamins & 61 & 3.5 & 17 & 1.6 & -54.3 & $<0.01$ \\
\hline
\end{tabular}

$A U R I=$ acute upper respiratory infection; $A L R I=$ acute lower respiratory infection; $C R D=$ chronic respiratory disease. $S D=$ standard deviation.

tion, mainly due to the reduction in the cost of antibiotic prescriptions.

The decrease in the average cost of drug prescriptions per patient in the impact survey also seemed to be related to the reduction in the mean cost of adjuvant drug prescription per patient (other than antibiotics, bronchodilator drugs or corticosteroids). Among the patients who received a bronchodilator prescription, the mean cost of bronchodilators per patient increased $(+33.0 \%)$ in the impact survey $(P<0.05)$; likewise, among patients who were prescribed corticosteroids, the mean 


\begin{tabular}{|c|c|c|c|c|}
\hline Variable & $\begin{array}{c}\text { Baseline survey } \\
\text { Mean (SD) }\end{array}$ & $\begin{array}{c}\text { Impact survey } \\
\text { Mean (SD) }\end{array}$ & $\%$ change $^{a}$ & $P$-value \\
\hline \multicolumn{5}{|l|}{$\begin{array}{l}\text { Cost of prescription for respiratory } \\
\text { patients prescribed drugs }(S P)\end{array}$} \\
\hline All respiratory patients & $205.6(138.8)$ & $151.7(111.0)$ & -26.2 & $<0.001$ \\
\hline AURI patients & $190.9(123.7)$ & $142.0(100.3)$ & -25.6 & $<0.001$ \\
\hline ALRI patients & $237.2(158.9)$ & $159.4(108.2)$ & -32.8 & $<0.001$ \\
\hline CDR patients & $258.1(190.0)$ & $220.9(160.0)$ & -14.4 & $>0.05$ \\
\hline Other patients & $178.1(97.6)$ & $99.4(58.4)$ & -44.2 & $<0.05$ \\
\hline $\begin{array}{l}\text { Cost of antibiotics for patients } \\
\text { prescribed drugs (SP) }\end{array}$ & $136.2(123.9)$ & 83.4 (99.9) & -38.8 & $<0.001$ \\
\hline \multicolumn{5}{|l|}{$\begin{array}{l}\text { Cost of antibiotics for patients } \\
\text { prescribed antibiotics }(S P)\end{array}$} \\
\hline All respiratory patients & $157.1(120.1)$ & $132.8(96.6)$ & -15.5 & $<0.001$ \\
\hline AURI patients & $149.6(107.1)$ & $127.9(91.8)$ & -14.5 & $<0.001$ \\
\hline ALRI patients & $174.3(147.3)$ & $145.1(94.2)$ & -16.8 & $<0.05$ \\
\hline CDR patients & $177.9(132.3)$ & $123.7(90.3)$ & -30.5 & $>0.05$ \\
\hline Other patients & $143.8(96.3)$ & $101.5(66.2)$ & -29.4 & $>0.05$ \\
\hline $\begin{array}{l}\text { Cost of bronchodilators for patients } \\
\text { prescribed bronchodilators }(S P)\end{array}$ & $74.2(72.1)$ & $98.7(84.0)$ & +33.0 & $<0.05$ \\
\hline $\begin{array}{l}\text { Cost of corticosteroids for patients } \\
\text { prescribed corticosteroids }(S P)\end{array}$ & $57.8(68.9)$ & $118.6(107.9)$ & +105.2 & $<0.01$ \\
\hline Cost of other drugs (SP) & $64.1(44.2)$ & $59.5(39.1)$ & -7.2 & $<0.01$ \\
\hline Total cost $(S P)$ & 356223 & 157182 & & \\
\hline$\%$ of total costs & $\%$ & $\%$ & & \\
\hline Antibiotics & 66.5 & 55.1 & -17.1 & $<0.001$ \\
\hline Bronchodilators & 2.7 & 4.7 & +74.1 & $<0.001$ \\
\hline Corticosteroids & 0.9 & 2.6 & +188.9 & $<0.001$ \\
\hline Other drugs & 29.9 & 37.6 & +25.8 & $<0.001$ \\
\hline
\end{tabular}

${ }^{a}$ Between impact and baseline surveys.

$S P=$ Syrian pounds; $A U R I=$ acute upper respiratory infection; $A L R I=$ acute lower respiratory infection; $C R D$ = chronic respiratory disease; $T B=$ tuberculosis; $S D=$ standard deviation .

cost of corticosteroids per patient increased $(+105.2 \%)(P<0.01)$.

The distribution of costs was significantly modified between the 2 surveys (Table 7). The cost of antibiotic prescriptions as a proportion of overall drug prescriptions decreased significantly $(-17.1 \%)$ in the impact survey at the expense of a significant increase in the proportion of bronchodilator, corticosteroid and other drug prescriptions.

\section{Discussion}

The focus of this study was not on its external validity or the generalizability of its findings. Our concern was to ensure the internal validity of the study, avoiding biases that would prevent comparability between the data sets of the baseline and impact surveys. Therefore we did not make a random selection of governorates, PHC facilities or 
GPs. In order to maximize comparability, both surveys used the same study protocol, were carried out within a 6-week interval (i.e. under similar weather and climate conditions) and involved the same 75 PHC facilities. As the GPs involved were the same, it was expected that a satisfactory level of comparability would be reached and only the behaviour of GPs in providing respiratory care would be different in the impact survey because of the training on PAL they received.

The number of respiratory patients registered in the impact survey was unexpectedly lower than in the baseline survey (39.1\% lower). The decrease was mainly observed in Homs (in 27 out of 30 PHC facilities) and Latakia (in 24 out of $28 \mathrm{PHC}$ facilities), but further investigation could not explain the reason for the decrease. On average, every GP managed clusters of 23.8 respiratory patients in the baseline survey and 14.5 in the impact survey. It is likely that patients included in the same cluster were managed in a similar manner by the GP. It is important to highlight that the observation unit for analysis was the patient and not the GP. Therefore, the results were likely to be influenced by some degree of clustering effect, although this may have been reduced by the relatively high number of GPs in the surveys. The clustering effect was likely to be higher in the baseline survey than in the impact survey because of the larger number of patients per cluster and this may have caused some distortion of comparability between the 2 data sets.

Nevertheless, in general the patients enrolled in the baseline survey were not significantly different from those recruited for the impact survey. This suggest that the 2 data sets were quite comparable.

More females attended the health facilities than males in both surveys. Similar results were reported in 8 countries where the first PAL surveys took place [1] and in studies conducted in Bolivia [7], Jordan [8], Kyrgyzstan [9] and Tunisia (A. Ben Kheder, personal communication).

In both surveys more than $20 \%$ of patients aged 5+ years who attended PHC facilities for any reason were respiratory patients. This proportion was $9 \%-37 \%$ in PAL surveys in 5 countries [1].

Feasibility tests of PAL using a similar study protocol carried out in Bolivia [7], Jordan (K. Abu Rumman, personal communication) and Kyrgyzstan [9] reported that training on PAL significantly reduced the referral of patients from primary to secondary care. In contrast, our study found that training on PAL significantly increased referrals from PHC units. Further studies are needed to explore whether and to what extent training on PAL reduces referral to higher levels of health care and therefore improves the integration of respiratory condition management into PHC services.

Our findings suggest that training on PAL promotes the use of SSE, as the rate of requests and the rate of smears performed increased between the 2 surveys. The proportion of respiratory patients eligible for SSE (symptom duration $\geq 15$ days) also increased. Similar results were reported in Bolivia [7] and Tunisia (A. Ben Kheder, personal communication). The proportion of respiratory patients who underwent SSE also significantly increased. The results did not show that applying PAL in PHC units increases TB detection because the sample size was too small to detect sufficient numbers of cases. A randomized controlled trial in South Africa showed a significant increase in TB case detection among respiratory patients after physicians had been trained on PAL [10]. In a feasibility study on PAL in Kyrgyzstan, the approach did not improve TB detection in terms of quantity and quality of cases [9]. However, the NTP of Kyrgyzstan was not involved in

المجلة الصحية لشرق المتوسط، منظمة الصحة العالمية، المجلد الخنامس عشر، العدد ب، 9 +. 
the development of PAL guidelines nor in the conduct of the study, whereas in Bolivia, Tunisia and the Syrian Arab Republic the NTP was fully involved in the various phases of PAL development. This suggests that involvement of the NTP in PAL adaptation and development is crucial in order to improve the management of suspected TB cases as an integral part of the management of respiratory conditions.

The survey clearly showed that applying PAL has an impact in reducing drug prescriptions in general through a reduction in the prescription of antibiotics and to some extent adjuvant drugs. It also showed a reduction in the cost of drug prescriptions per patient, particularly for antibiotics in acute respiratory infections. Similar results were reported in Bolivia [7], Jordan [8], Kyrgyzstan [9], Nepal [11] and Tunisia (A. Ben Kheder, personal communication). Our findings suggest that training on PAL tends to increase the prescription of inhaled medications. In general, these results indicate that PAL is likely to promote good management practices for asthma and chronic obstructive pulmonary disease. Similar results were observed in Algeria (N. Zidouni, personal communication), Jordan [8], South Africa [10], Kyrgyzstan [9] and Tunisia (A. Ben Kheder, personal communication).

In summary, our findings suggest that application of PAL improves quality in the selection of TB suspects, decreases drug prescriptions, particularly antibiotic prescriptions, increases the prescription of drugs for inhalation use and reduces the average cost of drug prescriptions in respiratory patients.
However, most of the results that have been reported so far, including ours, show only the short-term impact of PAL in the framework of pilot studies or operational research. Results of the long-term application of PAL are not yet available, but it is hoped that it will increase the proportion of respiratory patients managed in $\mathrm{PHC}$; decrease the proportion of respiratory patients hospitalized; improve TB screening among respiratory patients, particularly those who meet the definition of TB suspects; improve the quality of the diagnosis of pulmonary TB; decrease the number of asthma attacks and exacerbations of chronic obstructive pulmonary disease, particularly in emergency rooms; decrease the number of patients who are admitted to intensive care units for exacerbation of chronic respiratory diseases; reduce drug prescriptions for respiratory patients, particularly antibiotics; and reduce the cost of respiratory case management within the district health system. These, however, need to be carefully documented in countries such as El Salvador, Morocco and Kyrgyzstan, where PAL services have been provided on a routine basis in $\mathrm{PHC}$ settings for over 3 years.

\section{Acknowledgements}

The authors are indebted to Bashir Adib, Jamal Alouch, Mazin Kassibati, Muhamed Mussalamat, Muin Faluh, Naser Daâbul, Nadir Mameez and Yousser Muhamed. This study was supported by a grant from the World Health Organization.

\section{References}

1. Respiratory care in primary care settings-a survey in 9 countries. Geneva, World Health Organization, 2004 (WHO/ HTM/TB/2004.333).
2. The stop TB strategy. Building on and enhancing DOTS to meet the TB-related Millennium Development Goals. Geneva, 
World Health Organization, 2006 (WHO/ HTM/TB/2006.368).

3. Practical Approach to Lung health (PAL): a primary health care strategy for integrated management of respiratory conditions in people of five years of age and over. Geneva, World Health Organization, 2005 (WHO/HTM/TB/2005.351; WHO/NMH/ CHP/CPM/CRA/05.3).

4. Murray JF, Pio A, Ottmani S. PAL: a new and practical approach to lung health. International journal of tuberculosis and lung disease, 2006, 10:1188-91.

5. Practical Approach to Lung Health. Manual to initiate PAL implementation. Geneva, World Health Organization, 2009.

6. International statistical classification of diseases and related health problems, 10th revision. Geneva, World Health Organization, 2003.

7. Camacho $\mathrm{M}$ et al. Results of PAL feasibility test in primary health care facilities in four regions of Bolivia. International journal of tuberculosis and lung disease, 2007, 11:1246-52.

8. Abu Rumman $\mathrm{K}$ et al. Training on the Practical Approach to Lung Health: effect on drug prescribing in primary health care settings in Jordan. Eastern Mediterranean health journal, 2009, 15(1):111-121.

9. Brimkulov $\mathrm{N}$ et al. Feasibility test results of the Practical Approach to Lung health in Bishkek, Kyrgyzstan. International journal of tuberculosis and lung disease, 2009, 13(4):533-9.

10. Fairall LR et al. Effect of educational outreach to nurses on tuberculosis case detection and primary care of respiratory illness: pragmatic cluster randomized controlled trial. British medical journal, 2005, 331:750-4.

11. Shrestha $\mathrm{N}$ et al. Practical Approach to Lung Health in Nepal: better prescribing and reduction of cost. Tropical medicine and international health, 2006, 11:76572

\section{Fifth meeting of the Subgroup on Public-Private Mix for TB Care and Control (Cairo, Egypt, 3-5 June 2008)}

The Subgroup on Public-Private Mix (PPM) for TB Care and Control was established by the global Stop TB Partnership's DOTS Expansion Working Group to help promote and facilitate active engagement of all relevant public and private health care providers in TB control.

The fifth meeting to be hosted by the WHO Regional Office of the Eastern Mediterranean will concentrate on mechanisms and tools to building capacities of institutions supporting and/or undertaking TB care provision such as, national professional organizations, large hospitals, and corporate sector health establishments. Ways for effective use of International Standards for TB Care as the basis and a tool to achieve institutional strengthening for TB control will also be examined. Donor and community perspectives on PPM will be discussed and new initiatives such as PPM for TB/HIV and PPM MDR TB will be introduced.

المجلة الصحية لشرق المتو سط، منظمة الصحة العالمية، المجلد الخامس عشر، العدد ب، 9 . ب 